\title{
Scanning for presence in simple sentences is influenced by image value of nouns*
}

\author{
CARLTON T. JAMES and GLEN P. AYLWARD $\dagger$ \\ Rutgers-The State Liniversity, . Vew Brunswick, N.J. 08903
}

Ss were asked to scan transitive (A-N-V-A-N) sentences for the presence or absence of probe word. It took longer to locate abstract subject (object) nouns than to locate concrete subject (object) nouns.

Most research conducted under the memory scan paradigm (wherein $S$ decides whether a probe is a member of a previously designated memory set) has used digit materials. This is a natural choice if one's interest lies in a detailed specification of retrieval processes in primary memory, for the digits are, on the whole, a homogeneous set largely free of the bothersome problems of imagery and association value.

However, we think the scan paradigm can also be used profitably to examine how information is coded in primary memory. Some indication that the memory representations formed in this task contain semantic attributes is provided in a report by Kennedy \& Wilkes (1970). Using sentences, they obtained a flat serial position curve, with the exception of significantly faster RTs for subject and object noun probes. Wilkes \& Kennedy (1970) have proposed that their previous result reflected semantic rather than syntactic factors. Since all of the nouns in their previous study were concrete, image value (I value) is a likely candidate for the role of the operative semantic factor. To test this hypothesis, the present study used two types of sentences: HL sentences, containing high I-value subjects and low I-value objects, and LH sentences, containing just the opposite. If I value affects location time, an interaction between sentence type and noun function would be expected. If, however, some other semantic attribute associated with "nounness" was responsible for the Kennedy-Wilkes result, then location of subject (and object) probes should not depend upon I value.

\section{METHOD}

\section{Subjects and Design}

Sixteen Rutgers College underclassmen were paid $\$ 10$ to participate in the experiment. There were two Es. each of whom ran eight Ss. Two stimulus orders were used, and four of the Ss run by each $E$ received each order. Hence, there were two between-Ss variables, $E$ and order, and four $S$ s served for five sessions (a within-S variable) under each of these four conditions. There were two other within-S variables. sentence

*This research was supported in part by Grant 19859 from the National Institute of Mental Health and by a NIH Behavioral Sciences Support Grant to Rutgers University.

+Now at Fairleigh-Dickinson University. Requests for reprints should be addressed to Carlton T. James, 73 Easton Ave., Psychology Department. Rutgers University, New Brunswick N.J. 08903 . type (two levels) and position (five levels) in the sentence. Therefore, the design was a 2 by 2 by 4 by 5 by 2 by 5 factorial.

\section{Materials}

A total of 36 sentences was used in the experiment, all having the basic form Adj-N-V-Adj-N. (Some sestences had articles, others did not: only the five content words were used as probes.) One objection to the results reported by Kennedy and Wilkes is that they repeatedly probed only three highly overlearned sentences. As Sternberg (1969) has obtained the same results with fixed and variable memory ensemble methodologies (with digits), the repeated testing of the same sentences is probably not critical. However, it is quite possible that the six nouns chosen by Kennedy and Wilkes might have been in some sense atypical. The use of 36 sentences thus extends the generality of their result.

Half the sentences (HL) had a high I-value subject and a low I-value object (e.g., "The retreating army abandoned the temporary bivouac"). The remaining 18 sentences ( $\mathrm{LH})$ had a low I-value subject and a high I-value object (e.g., "His continued insolence angered the friendly islander"). High I-value nouns had a mean rating (Paivio, Yuille, \& Madigan, 1968) of 6.00 vs 3.30 for low I-value nouns. Mean frequency was approximately equal for both sets of nouns and verbs; adjectives were somewhat less frequent, by the Kučera and Francis (1967) nouns.

\section{Procedure}

Each session began with two practice trials, followed by 100 test trials. Of these, 72 required a "yes" response, and the remaining 28 tests consisted of presenting a probe word which was not contained in the original sentence. The sentences used on "catch" trials varied from session to session. The order of "yes" trials and "catch" trials was random in each session. For the 72 "yes" trials, the number of times that each position was tested on a particular day was randomly determined, under the constraint that, across all 5 days, each sentence had each of its five content words tested twice. Hence, overall, each position in $\mathrm{HL}$ and $\mathrm{LH}$ sentences was tested 36 times for each S.

Five blocks of sentences were recorded on tape in two orders, 1-5 and 5-1, with a $20-\mathrm{sec}$ silent interval and a ready signal preceding each sentence. The sentences were presented over earphones. and half the Ss received each order. Approximately $3 \mathrm{sec}$ following presentation of a sentence, $E$ turned on tachistoscope, thereby illuminating the probe word. The $S$ would then communicate his decision by pushing one of two buttons (indicating whether or not he judged the probe word to have been contained in the memory set). This response turned off the light in the tachistoscope and stopped a millisecond timer. Directions stressed both speed and accuracy.

\section{RESULTS AND DISCUSSION}

The error rate was $2.3 \%$ for "yes" trials and $1.7 \%$ for "no" trials; these RTs were excluded from analysis. Mean RT for position as a function of sentence type is presented in Table 1. Analysis revealed the only significant main effects to be sessions. $F(4.48)=32.97$. $\mathrm{p}<.01$. and position. $\mathrm{F}(4.48)=8.13, \mathrm{p}<.01$. Statistical significance was attained by two interactions not viewed as theoretically important: Session by Position. $F(16.192)=2.39 . p<.01$ : and $E$ by Session by Sentence Type, $F(4.48)=2.90 . p<.05$.

The predicted interaction between sentence type and 
Table 1

Mean Latency (in Milliseconds) as a Function of Sentence Type and Position in the Sentence

\begin{tabular}{cccccc}
\hline & \multicolumn{5}{c}{ Position } \\
\cline { 2 - 6 } Sentence \\
\cline { 2 - 6 } Type & Adjective & Noun & Verb & Adjective & Noun \\
\hline HL & 780 & 746 & 808 & 792 & 763 \\
LH & 798 & 799 & 799 & 784 & 738 \\
\hline
\end{tabular}

position was highly reliable. $\mathrm{F}(4,48)=9.58 . \mathrm{p}<.01$ However. this interaction was not uniformly realized across sessions, yielding a statistically significant three-way interaction. $F(16.192)=2.11 . p<.01$. Analysis of the simple two-way interactions revealed that the Sentence Type by Position interaction was reliable (every $p<.01$ ) for all but Session 4 $(.10>p>.05)$. In all sessions, location of subject nouns was faster for HL than for LH sentences, and location of object nouns was faster for LH than for HL sentences (see Table 2). The three-way interaction thus reflects differing magnitudes of the Sentence Type by Position interaction as a function of sessions, but the nature of the interaction is basically the same. Consequently, simple main effects were calculated for the overall two-way interaction.

The primary empirical hypotheses of this study were that RT for nonnouns would not differ as a function of sentence type, that RT to the subject noun would be

Table 2

Mean Difference Scores (in Milliseconds) for Subject and Object Nouns as a Function of Session

\begin{tabular}{lcrccc}
\hline & \multicolumn{5}{c}{ Session } \\
\cline { 2 - 6 } & 1 & 2 & 3 & 4 & 5 \\
\hline Subject* & 94 & 68 & 57 & 17 & 30 \\
Object** & 28 & 2 & 35 & 20 & 39 \\
\hline
\end{tabular}

${ }^{*} L H \cdot H L \quad *{ }^{*} H L \cdot L H$ faster for HL sentences, and that RT would be faster for LH object nouns. Tests for simple main effects confirmed these predictions; significant comparisons were obtained for subject nouns, $F(1.48)=29.08$, $\mathrm{p}<.01$. and for object nouns, $\mathrm{F}(1.48)=6.28 . \mathrm{p}<.05$, but not for any other position (every $\mathrm{p}>.05$ ).

Clearly. high I value nouns from both $\mathrm{HL}$ and $\mathrm{LH}$ sentences were located faster than any other word from either sentence. It would thus appear that I-value is the semantic factor responsible for faster location of nouns in the memory scan paradigm.

These data also have implications for the controversy over the nature of coding in primary memory. If, in this task. primary memory is being scanned, then imaginal and semantic information must be registered in primary memory as well as in secondary memory. Such results, demonstrating a continuity in terms of coding characteristics, weaken the theoretical value of dichotomizing (postsensory) memory into separate stores.

\section{REFERENCES}

Kennedy, R. A., \& Wilkes, A. L. A comparison of retrieval and location operations in active and passive sentences. British Journal of Psychology, 1970, 61, 491-498.

Kucera, H., \& Francis, W. N. Computational analysis of present-day American English. Providence, R.I: Brown University Press, 1967.

Paivio, A., Y uille, J. C., \& Madigan, S. A. Concreteness, imagery, and meaningfulness values for 925 nouns. Journal of Experimental Psychology, 1968, Monograph Supplement, No. 1, Part 2.

Sternberg, S. Memory scanning: Memory processes revealed by reaction-time experiments. American Scientist, 1969, 57 , 421-457.

Wilkes, A. L., \& Kennedy, R. A. The relative accessibility of list items within different pause-defined groups. Journal of Verbal Learning \& Verbal Behavior, 1970, 9, 197-201.

(Received for publication June 18, 1973.) 